\title{
La ciencia política en Colombia en el 2005-2006. E1 análisis del conflicto armado y de las elecciones, dos temas tradicionales, pero con enfoques nuevos
}

\author{
DAVID GARIBAY ${ }^{1}$
}

Los temas de interés de las ciencias sociales en Colombia, y en particular de la ciencia política y la sociología, están fuertemente marcados por la evolución del proceso socio-político propio de este país, así como por la particular coexistencia entre la continuidad de las instituciones representativas y la permanencia de un conflicto armado interno de gran dimensión. Las elecciones programadas en el 2006 (parlamentarias en marzo y presidenciales en mayo), así como la perspectiva de la reelección de Álvaro Uribe, quien ha mantenido una posición rígida respecto al conflicto armado, han llevado a poner nuevamente en el centro de las preocupaciones académicas tanto el análisis de las instituciones, y en particular de las elecciones, como el análisis del conflicto interno.

La forma en que se han estudiado recientemente en Colombia estos dos temas hace resaltar un fenómeno algo paradójico: aunque estén cada vez más entrelazados, el estudio de cada uno de ellos parece seguir lógicas autónomas, en las cuales uno no parece ser un determinante importante del otro. De forma más precisa, se puede considerar que por una parte, los análisis del comportamiento del electorado colombiano no contemplan como un factor decisivo la coyuntura del conflicto interno, y por la otra, el estudio de la confrontación entre los actores armados parece seguir independiente de la evolución de las políticas gubernamentales. Esta situación específica de las ciencias sociales refleja a su manera un análisis más general que se puede realizar acerca de la situación política del país, como lo ha demostrado desde hace ya varios años el sociólogo francés Daniel Pécaut en la reedición actualizada de sus inestimables crónicas y ensayos sobre la vida política colombiana (Pécaut, 1988; 2006).

Cabe resaltar que el estudio de la violencia y el análisis del comportamiento electoral son temas que han significado un reconocimiento internacional para los académicos colombianos, desde hace varios años, por la originalidad de sus planteamientos y de sus investigaciones. De hecho, en los trabajos publicados en los últimos meses, se perciben fuertes continuidades con los trabajos anteriores, pero se pueden destacar también ciertas novedades en su tratamiento, en particular la introducción de la doble dinámica de la internacionalización y de

1 Profesor titular de ciencia política, Facultad de Derecho y Ciencia Política, Universidad Lumière Lyon 2, Francia. Correo electrónico: david.garibay@univ-lyon2.fr. Agradezco la valiosa ayuda bibliográfica brindada por Juan Felipe Carrillo, doctorando en ciencia politica del Institut d'Etudes Politiques de Lyon (Francia). 
la afirmación de identidades individuales específicas en los procesos políticos y sociales nacionales ${ }^{2}$.

\section{Las principales contribuciones de los últimos años y sus orientaciones generales}

Tres publicaciones recientes permiten observar la importancia que adquieren los dos campos de reflexión señalados anteriormente a la hora de hacer un balance del primer período presidencial de Álvaro Uribe (2002-2006). La revista Análisis Político, publicada por el Instituto de Estudios Políticos y Relaciones Internacionales $^{3}$, dedicó un número entero ${ }^{4}$ a los "Balances y perspectivas de 4 años del gobierno Uribe". De los seis artículos, tres abordan un aspecto específico del conflicto armado y uno el sistema de partidos. Por otra parte, es sintomático que el libro colectivo dirigido par Fernando Leal Buitrago ${ }^{5}$, titulado En la encrucijada, Colombia en el siglo XXI (2006a), renueve una tradición de contribuciones académicas sobre la coyuntura nacional e incluya artículos que tratan del sistema electoral y de partidos en cada una de sus tres secciones (actores institucionales, actores sociales, políticas públicas). También incluye artículos acerca de los actores del conflicto y de las políticas de paz o de guerra. El tercer ejemplo corresponde al libro colectivo dirigido por el politólogo Francisco Gutiérrez Sanín y el historiador Gonzalo Sánchez, ambos del IEPRI, Nuestra guerra sin nombre (2006) dedicado principalmente a analizar diversos aspectos del conflicto armado. Este trabajo contiene un gran número de contribuciones acerca de los actores y las dimensiones del conflicto, pero concentra también parte del análisis en el Estado colombiano y en el régimen político en un contexto de guerra.

En los últimos meses, la preponderancia del análisis de las elecciones por una parte, y del conflicto armado por la otra, ha llevado a reducir la importancia acordada a los trabajos acerca de otros temas. Sin embargo, en otras áreas de la ciencia política, se han publicado importantes contribuciones, en particular sobre políticas públicas: por ejemplo Luis Mauricio Cuervo, economista de la Universidad Externado, publicó un estudio sobre la aplicación de la política de justicia (Cuervo, 2005) y participó en un trabajo con sociólogos y geógrafos franceses

2 En el marco de una reseña limitada a las últimas publicaciones en ciencia política en Colombia, no es posible presentar el conjunto de las orientaciones de la producción académica de esta disciplina. Por lo tanto, solamente se señalarán algunas producciones anteriores, con el fin de mostrar las novedades en los trabajos más recientes. Para algunas reseñas generales y recientes sobre la ciencia política en Colombia, véase Bejarano, Wills (2005), y sobre las ciencias sociales de forma más general: Leal Buitrago, Rey (2000). Sobre los enfoques del estudio de la violencia, véase Pécaut (1998).

3 El IEPRI, perteneciente a la Universidad Nacional de Colombia, es el centro de investigaciones en ciencias sociales con más trayectoria en Colombia. Fue creado hace 20 años, y desde su creación sus investigadores han aportado contribuciones muy significativas al estudio de la violencia y del conflicto armado, mediante el análisis de los diferentes actores que participan de él.

$\mathrm{N}^{\circ} 57$, mayo-julio 2006.

5 Sociólogo del IEPRI. 
sobre la metropolización de Bogotá que se sitúa, de forma original, en la intersección entre geografía urbana y ciencia política (Gouëset, Cuervo; Lulle, Coing, 2005). La Universidad Externado también ofrece una publicación anual acerca del análisis de las políticas públicas. El número del año pasado estuvo dedicado tanto a las políticas municipales como al tema de la responsabilidad social de las empresas (Observatorio de politicas, ejecución y resultados de la administración pública, OPERA, $\mathrm{CIPE}^{6}$, vol. 5, 2005).

\section{El análisis del conflicto colombiano desde el punto de vista nacional: continuidades y nuevos enfoques}

Con respecto al análisis del conflicto colombiano, los politólogos de este país se destacan por la continuidad de sus análisis respecto a la manera de entenderlo, bajo una perspectiva que busca mostrar las relaciones entre las estrategias de los actores en presencia y las consecuencias sociales de la violencia sobre el conjunto de la sociedad. En efecto, el análisis detallado de la evolución propia de los actores del conflicto armado, ha sido una constante de las ciencias sociales en Colombia desde los primeros trabajos académicos que se han interesado en el tema ${ }^{7}$. Las producciones recientes presentadas en esta reseña se sitúan en parte en esta continuidad. Los análisis de las orientaciones de la política gubernamental actual en materia de paz y/o de guerra comparan la acción del primer gobierno de Uribe con sus predecesores, desde una perspectiva de análisis - presente en Colombia desde los años 1980- que considera la política de paz como una política pública específica. Por ejemplo, Mauricio García, politólogo del CINEP ${ }^{8}$, actualiza el largo trabajo que ha emprendido desde hace varios años acerca de las políticas de paz de los diferentes gobiernos (García, 2006a). Por su parte, Francisco Leal Buitrago muestra que detrás de la aparente unidad y coherencia de la política del Presidente Uribe en materia de "seguridad democrática", existe tanta improvisación como en los gobiernos anteriores (Leal Buitrago, 2005, 2006b, 2006c). Finalmente, Armando Borrero, sociólogo de la Universidad Nacional, completa este panorama

$6 \quad$ El Centro de Investigaciones y Proyectos especiales, CIPE, de la Universidad Externado de Colombia es un centro de investigación. Se creó en 1993, y se especializó en las áreas de políticas públicas y de relaciones internacionales.

7 Probablemente el primer trabajo académico sobre la violencia en Colombia es el de Guzmán Campos, Fals Borda y Umãna Luna, del año 1962. En esta obra pionera, los autores ponían especial atención en el análisis de los actores de la violencia. Desde entonces, esta perspectiva ha sido ampliada, según las épocas, a la guerrilla, los narcotraficantes, los paramilitares y/o el ejército. Incluso en la década de 1980, cuando se abrieron otros debates sobre el estudio de la violencia a partir de un enfoque centrado más en el fenómeno de la violencia como fenómeno social que en el comportamiento de determinados actores, no se dejó de lado el análisis de las trayectorias de cada una de las organizaciones armadas (Comisión de Estudios sobre la Violencia, 1987).

8 El Centro de Investigación y Educación Popular (CINEP) es un centro jesuita de gran trayectoria, que ha orientado parte de sus actividades al estudio del conflicto armado, en particular en sus dimensiones locales, así como a los procesos de paz. 
gubernamental con un análisis de la evolución interna de las fuerzas armadas, en el contexto específico de su profesionalización, implementada de forma reciente, y del aumento de sus recursos, correspondiente a la ayuda norte-americana mediante el Plan Colombia (Borrero, 2006).

Este análisis de los actores gubernamentales tiene su contraparte en las reflexiones acerca de la evolución de la situación de las guerrillas y de sus estrategias por reconocidos especialistas de las guerrillas colombianas, ya sea en una perspectiva comparada sobre los dos grupos de guerrilla activos (Fuerzas Armadas Revolucionarias de Colombia, FARC, y Ejército de Liberación Nacional eLN), como Camilo Echandía, economista de la Universidad Externado de Colombia, y Román Ortiz, politólogo de la Universidad de los Andes (Echandía, 2006a, 2006b; Ortiz, 2006). También existen trabajos acerca de un grupo en particular, como es el caso del estudio de Eduardo Pizarro, politólogo del IEPRI, sobre las FARC (Pizarro, 2006) o de Mario Aguilera, sociólogo de la Universidad Nacional de Colombia sobre el ELN (Aguilera, 2006). El análisis comparado de estos actores violentos ha sido completado desde una década por trabajos dedicados a los actores que han tomado cada vez más importancia en el conflicto interno, como los narcotraficantes y los grupos paramilitares. En esta línea, Francisco Thoumi, economista de la Universidad del Rosario, ha actualizado sus trabajos sobre la producción y tráfico de drogas, con una comparación del desarrollo histórico de la producción de drogas en Colombia y Afganistán (Thoumi, 2005). Por su parte, Ricardo Vargas, sociólogo de la Universidad Nacional de Colombia, ha mostrado la importancia de la economía de la droga en el conflicto armado, y en particular de la orientación de la acción armada contra las drogas (Vargas, 2005). Finalmente, el proceso de negociación y de desarme que llevó a cabo el gobierno de Álvaro Uribe con determinados grupos paramilitares contribuyó a enfocar varios trabajos sobre este último actor, como en el caso del libro dirigido por Alfredo Rangel (economista y politólogo de la Fundación Seguridad y Democracia; Rangel, 2005), o de las reflexiones de Carlos Medina (politólogo de la Universidad Nacional de Colombia; Medina, 2005) o de Ingrid Johanna Bolívar (politóloga del CinEP; Bolívar, 2005).

Muchos trabajos académicos señalan desde los años 90 lo difícil que es analizar a actores armados que tienen al mismo tiempo una influencia creciente en el escenario político colombiano y una existencia formal mucho menos fija que antes, pues las relaciones que mantienen entre ellos son cada vez más complejas y fluctuantes. Esta característica ha llevado a los analistas a reflexionar acerca de la confusión que se genera entre actores, tanto en sus relaciones mutuas como en los efectos que produce el uso que hacen de la violencia en la sociedad colombiana. En este aspecto indagan en particular Mauricio Romero, politólogo del IEPRI y Álvaro Camacho, sociólogo de la Universidad de los Andes, en un libro acerca de las relaciones entre los paramilitares y los carteles de la droga (Romero, 2006; Camacho, 2006). En una perspectiva innovadora, Juana Schlenker, antropóloga de la Universidad de los Andes y Manuel Iturralde, abogado de la Universidad de los Andes, analizan cómo la retórica sobre los derechos humanos ha sido transformada en un recurso usado por todos los actores del conflicto. Este abuso de 
la referencia, y la imposición de visiones parciales, tiende a limitar el carácter general de las acciones, discursos y movilizaciones sobre los Derechos Humanos que podrían tener un efecto pacificador (Schlenker, Iturralde, 2006).

Cabe subrayar además que esta confusión no opera solamente entre actores ilegales. En un trabajo comparativo acerca del conjunto de los protagonistas armados, legales e ilegales, Fernando Cubides, sociólogo de la Universidad Nacional de Colombia, muestra que existen comportamientos similares en lo que denomina con cierta ironía las "burocracias armadas" (Cubides, 2005b). En efecto, el proceso ya mencionado de negociación entre el gobierno de Álvaro Uribe y determinados grupos paramilitares (en particular las Autodefensas Unidas de Colombia, $\mathrm{AUC}^{9}$ ) ha sido el objeto de numerosos estudios recientes, que analizan el tipo de relación que ha imperado entre las fuerzas del Estado y los grupos paramilitares. Fernando Estrada, especialista en comunicación social de la Universidad EAFIT de Medellín (Estrada, 2006), así como Francisco Gutiérrez Sanín y Mauricio Barón, politólogos del IEPRI (Gutiérrez, Barón, 2006) privilegian un enfoque territorial para analizar este tema. Su meta consiste en evidenciar las formas de control local que ejercen algunos grupos paramilitares de forma paralela a las estructuras locales del Estado o en ausencia de éstas.

En un análisis más histórico de las relaciones entre el gobierno y las AUC, Daniel García-Peña, politólogo de la Universidad Nacional de Colombia, muestra cómo éstas han evolucionado desde la creación de estos grupos en los años 1980 hasta la decisión de entablar un proceso de negociación (García-Peña, 2005). Por su parte, Fernando Cubides se centra particularmente en el análisis del proceso de negociación como tal, para mostrar cómo se llegó a un arreglo, en el cual se concedió de facto una amnistía a los grupos paramilitares, a pesar de las exacciones cometidas contra la población civil, pues el acuerdo prevé que los responsables de los grupos sean investigados por actos graves de violaciones a los derechos humanos, pero con límites temporales muy cortos como para realizar investigaciones jurídicas completas (Cubides, 2005a; Cubides, 2006).

De manera más global, el enfoque privilegiado en el análisis del conflicto armado en Colombia se centró primero en la evolución de los actores colectivos nacionales, y de forma más reciente en la interpretación de la violencia como fenómeno social integral, que tiene que ser descifrado a la luz de un complejo proceso de construcción social y política nacional. Esta perspectiva ha llevado durante mucho tiempo a dejar de lado el análisis de tres otras dimensiones del conflicto: la perspectiva de las víctimas, las experiencias personales e individuales del conflicto, y finalmente los aspectos internacionales, siendo el tercer aspecto el más trabajado en los últimos años.

9 Los grupos paramilitares en Colombia se caracterizan por no tener una existencia formalizada como organizaciones. Sin embargo, en 1997, se crean las AUC como un intento por parte de su dirigente de la época, Carlos Castaño, de reunir varios grupos regionales y de aparecer como una agrupación política. En 2003, se entablan negociaciones con el gobierno, las que desembocarán en un acuerdo de paz y el desarme de este grupo. 
Primero, las víctimas del conflicto armado siguen siendo una temática poco examinada en las ciencias sociales colombianas. En las publicaciones consultadas, que tratan de forma abundante del conflicto armado, no se registra en el período 2005-2006 una sola contribución que trate directamente de las víctimas del conflicto, ya sea sobre las movilizaciones en torno a los secuestrados, o sobre un tema particularmente candente, como es el de los desplazados internos por la violencia ${ }^{10}$. Sin embargo, el trabajo de Mauricio García -mencionado anteriormente- sobre las movilizaciones sociales y colectivas por la paz, analizadas como un movimiento social, constituye un aporte original e importante en este aspecto (García, 2005, 2006b). En lo relativo a las dimensiones subjetivas y personales del conflicto, acerca de la forma en que la violencia afecta y modifica las identidades personales, y por lo tanto colectivas, se destaca un trabajo reciente sobre la experiencia personal de los desmovilizados, de Kimberly Theidon, antropóloga, y Paola Betancourt, ambas del Instituto Praxis de la Universidad de Harvard (Theidon, Betancourt, 2006).

Desde la perspectiva de género, María Emma Wills, politóloga de la Universidad de los Andes, propone un trabajo muy novedoso en su enfoque, al estudiar cómo la participación de las mujeres en actividades combatientes modifica el rol social al cual han sido asignadas tradicionalmente (Wills, 2005a). A su vez, desde la perspectiva de los estudios de minorías, el trabajo de Carlos Agudelo, (sociólogo del Institut des Hautes Études d'Amérique Latine de París) sobre las comunidades negras en Colombia, muestra cómo la identidad colectiva de una minoría étnica se construye en un primer momento sobre la base de una situación de discriminación y de afirmación cultural, y cómo este proceso de construcción ha sido desestructurado de forma reciente por la violencia en las zonas de presencia de comunidades negras (Agudelo, 2005).

\section{Novedades del análisis del conflicto colombiano en los últimos tiempos: la introducción de una perspectiva internacional más sistemática}

Como comentamos anteriormente, y sin negar la importancia del análisis sociológico acerca de la evolución de los actores armados dentro del escenario nacional, varios académicos colombianos han introducido la variable internacional, lo que puede ser considerado como un aporte significativo en la ciencia política colombiana en estos últimos años. Este enfoque fue iniciado con el interés manifestado por Estados Unidos hacia el conflicto colombiano y confirmado posteriormente

10 Esto no significa que estos temas no sean tratados por la ciencia política colombiana, sino que no se ha visto en las publicaciones consultadas algún trabajo reciente sobre ellos, lo que puede ser asumido como una limitación de esta reseña si fuese el caso. 
por la puesta en marcha del Plan Colombia ${ }^{11}$. El análisis académico sobre esta dimensión del conflicto abarcó desde el principio de la década del 90 los efectos de la política norteamericana en dos aspectos: los comportamientos de los actores en presencia en el conflicto y la inserción internacional de Colombia, a través del calificativo polémico de "país problema". El hecho que en el libro Nuestra guerra sin nombre coordinado por Francisco Gutiérrez Sanín y Gonzalo Sánchez, la primera parte esté dedicada a la internacionalización del conflicto, cuando esta dimensión estaba casi ausente en los libros colectivos publicados sobre el tema hasta mediados de la década del 90 , es sintomático de la importancia que se empieza a acordar a esta dimensión. Sigue de hecho siendo el objeto de un minucioso trabajo de parte de varios cientistas sociales, en particular Diana Rojas, politóloga del IEPRI, sobre la influencia de Estados Unidos en el conflicto (Rojas, 2006a, 2006b), o Arlene Tickner, politóloga de la Universidad de los Andes, sobre la visión de Estados Unidos acerca de la seguridad internacional y el conflicto colombiano (Tickner, 2006). En la misma línea, se puede señalar el trabajo de Rodrigo Pardo, politólogo, ex-canciller, y ahora director de la Revista Semana, sobre los efectos del conflicto en la inserción internacional de Colombia (Pardo, 2006).

Además, la variable internacional se ha visto completada por estudios que centran su atención en otros actores estatales internacionales, en particular Europa y los países vecinos de Colombia, como lo hace Socorro Ramírez, politóloga del IEPRI (Ramírez, 2006a, 2006b), quien ha sido una de las investigadoras más prolíficas acerca de la variable internacional en el análisis del conflicto (Ramírez, 2004). Ha contribuido también a reorientar esta visión no solamente desde Estados Unidos, sino también desde la posición de los países de la región (Ramírez, 2006c) y en particular de Venezuela (Ramírez, Cárdenas, 2005) y Brasil (Ramírez, Cepik, 2005). De hecho, el interés por el estudio de la posición de los países vecinos de Colombia en el conflicto parece generalizarse: por ejemplo, en esta visión de un conflicto con consecuencias regionales, es interesante leer el número 60 de la revista Colombia Internacional dedicado a las "Percepciones hemisféricas de la crisis colombiana", en el cual se presentan visiones desde Brasil, Perú o Ecuador.

Esta mirada tiene también como consecuencia un aumento del número de publicaciones centradas en la evolución de los países vecinos y de sus relaciones con Colombia, como es el caso del trabajo de Julie Massal, politóloga del IEPRI, sobre Ecuador (Massal, 2006). Es notable que una parte importante de los artículos de la edición 2005/2006 del anuario OAsis (Observatorio de Análisis de los Sistemas Internacionales, CIPE, Universidad Externado de Colombia) también estén dedicados al estudio de la política exterior colombiana, con énfasis en las relaciones con los países vecinos o en estudios sobre la coyuntura política de países

11 Pensado inicialmente por el Presidente Andrés Pastrana (1998-2002) en 1998 como una ayuda financiera internacional a Colombia para luchar contra el tráfico de drogas, el Plan Colombia fue lanzado en 2000. Se trata de un plan de ayuda militar de parte de Estados Unidos hacia Colombia, que ha permitido reforzar la capacidad y los recursos del ejército y de la policía contra el tráfico de drogas y contra la guerrilla. 
como Ecuador y Bolivia. Este interés por la región es significativo en un anuario que se ha caracterizado desde su creación por ser una de los pocas publicaciones del país especializada en temas internacionales. Como límite, cabe señalar sin embargo que esta extensión de la mirada sobre la internacionalización del conflicto ha estado por el momento orientada principalmente hacia actores estatales o supra estatales (como la Unión Europea). La dimensión transnacional del conflicto colombiano, mediante el análisis del tráfico de drogas o de la situación de los refugiados en países vecinos, o el papel de los actores no estatales (en particular de las ONG) parece ser hasta ahora una dimensión marginal en el estudio reciente de las dimensiones de internacionalización del conflicto armado en Colombia.

\section{Un análisis electoral y del sistema de partidos desconectado del conflicto}

El otro tema privilegiado en la ciencia política colombiana en el 2006 ha sido el análisis electoral. Como en el caso del estudio del conflicto armado, los enfoques tradicionales han sido enriquecidos por cuestionamientos nuevos. En este campo, se produce también la desconexión señalada anteriormente para el estudio del conflicto armado, pues ambos temas parecen evolucionar de forma autónoma el uno del otro. Los especialistas del tema han propuesto varias interpretaciones de la evolución del sistema de partidos colombiano y en particular de la desagregación de las identidades bipartidistas, tanto a través del comportamiento electoral de los colombianos, como a través de la evolución de los partidos. Llama la atención que con enfoques y metodologías diferentes, muchos de los trabajos publicados en este ámbito muestran una fuerte autonomía con relación al conflicto armado.

Diana Hoyos, politóloga del IEPRI, insiste en la importancia de los niveles locales para entender la estabilidad de un sistema de partidos que ha experimentado una fuerte fragmentación desde los años 90 (Hoyos, 2006). Por su lado, al analizar los modelos de comportamiento electoral en Colombia con métodos cuantitativos, Gary Hoskin, Rodolfo Masias y Marcela Galvis, quienes son politólogos de la Universidad de los Andes ${ }^{12}$, demuestran que los elementos contextuales (como el conflicto) no son elementos explicativos centrales en la decisión del voto. Impera más bien el perfil de los candidatos o los temas de campaña (Hoskin, Masias, Galvis, 2005). En un plano más partidista, Francisco Gutiérrez muestra que la novedad del sistema de partidos que ha surgido durante el primer mandato de Álvaro Uribe radica en el papel central asumido por el Presidente de la República. En los mandatos anteriores, los parlamentarios, cualquiera sea su partido, constituían grupos informales que apoyaban al Ejecutivo o limitaban su trabajo. En este período, es el Presidente quien tiene la voluntad de reestructurar el régimen de partidos para que

12 El Departamento de Ciencia Política de esta universidad es uno de los principales centros académicos especializados en el análisis del comportamiento electoral en Colombia. 
emerjan formaciones partidarias claramente identificadas por su apoyo $\mathrm{u}$ oposición a su proyecto (Gutiérrez, 2006). A cambio, en un análisis más centrado en los equilibrios institucionales que en el régimen de partidos, Pedro Medellín, politólogo del Instituto Universitario Ortega y Gasset de Madrid, critica la hipótesis de un reforzamiento de la figura presidencial, al analizar en detalle la reducción de los márgenes de acciones reales del Ejecutivo (Medellín, 2006).

Sin embargo, en los trabajos acerca del comportamiento electoral de los colombianos, aparecen dos tipos de enfoques nuevos, que reflejan la importancia de la dimensión internacional y la emergencia de nuevas referencias en términos de identidades colectivas. El primero de estos enfoques, el transnacional, se interesa especialmente en la migración a Estados Unidos y la creación de identidades políticas específicas por parte de los migrantes colombianos en América del norte. Cristina Escobar, politóloga de la Temple University de Filadelfia, muestra que los inmigrantes colombianos en Estados Unidos son actores políticos activos tanto en su sociedad de origen como en la que los recibe, sobre todo cuando obtienen la doble nacionalidad. Subraya el papel central de las asociaciones de migrantes en este proceso de creación de una doble ciudadanía (Escobar, 2005). De paso, Escobar corrobora estudios anteriores sobre la participación política de los emigrantes en Estados Unidos o de emigrantes a Europa.

Otro enfoque novedoso en el ámbito del comportamiento electoral corresponde a la incorporación reciente de una perspectiva de género ya mencionada anteriormente, tanto en los análisis electorales como en los estudios de las movilizaciones sociales. El trabajo de María Emma Wills sobre la historia del sufragio femenino en Colombia y de la extensión del sufragio y de la ciudadanía (Wills, 2005b), es prolongado por otro estudio, de Angélica Bernal Olarte, politóloga del IEPRI, sobre los obstáculos que las mujeres enfrentan a la hora de participar en la vida de los partidos políticos (Bernal Olarte, 2006). En relación con esta renovación de los estudios electorales a partir de la perspectiva de género, Julie Massal introduce una perspectiva comparada en su reseña del libro de Margarita León sobre la aplicación de cuotas en la región andina (Massal, 2005). Este enfoque ha sido aplicado también a las movilizaciones sociales, en una innovadora contribución de María Emma Wills y Diana Gómez Correal acerca del surgimiento y el desarrollo de los movimientos sociales de mujeres en Colombia (Wills, Gómez Correal, 2006). Cabe señalar que esta contribución es una de las primeras sobre los movimientos de mujeres, no solamente en los libros de análisis que versan sobre la coyuntura nacional, sino también de forma más general en las ciencias sociales colombianas.

\section{Bibliografía}

Agudelo, Carlos. 2005. Multiculturalismo en Colombia: política, inclusión y exclusión de poblaciones negras. Medellín: La Carreta Editores.

Aguilera, Mario. 2006. "El ELN entre armas y política". En Gutiérrez Sanín, Francisco, Sánchez, Gonzalo, Nuestra guerra sin nombre. Bogotá: Norma, 208-266. 
Bejarano, Ana María; Wills, María Emma, 2005, "La ciencia política en Colombia, de vocación a disciplina". Revista de Ciencia Politica 25 (1): 111-123.

Bernal Olarte, Angélica. 2006. “QQué es ganar y qué es perder en política?: los retos en la participación electoral". Análisis Politico 56: 72-92.

Borrero, Armando. 2006. "Los militares: dolores del crecimiento". En Leal Buitrago, Fernando. 2006a, En la encrucijada, Colombia en el siglo XXI. Bogotá: Norma, 113-146.

BolíVAR, INGRID JoHANNA. 2005. "Las AUC como una formación elitista: normatividad social, legitima defensa y producción de diferencias”. Controversia 185: 51-88.

Camacho, Álvaro. 2006. "De narcos, paracracias y mafias". En Leal Buitrago, Fernando. 2006a. En la encrucijada, Colombia en el siglo XXI. Bogotá: Norma, 387-421.

Comisión de Estudios sobre la Violencia. 1987. Colombia: violencia y democracia. Bogotá: Universidad Nacional.

Cubides, Fernando. 2005a. "Santa Fe de Ralito: avatares e incongruencias de un conato de negociación". Análisis Político 53: 3-21.

Cubides, Fernando. 2005b. Burocracias armadas. Bogotá: Norma.

Cubides, Fernando. 2006a. "Proceso inconcluso, verdades a medias: para un balance de las negociaciones del gobierno Uribe con los paramilitares". Análisis Político 57: 55-64.

Cuervo, Luis Mauricio. 2005. Lineamientos de politica pública para el acceso a la justicia de personas de escasos recursos. Bogotá: Universidad Externado de Colombia.

EChandía, CAmilo. 2006a. "Conducta de la guerrilla durante el gobierno Uribe Vélez: de las lógicas de control territorial a las lógicas de control estratégico". Análisis Político 57: 31-54.

Echandía, Camilo. 2006b. Dos décadas de escalamiento del conflicto armado en Colombia (1986-2006). Bogotá, Universidad Externado de Colombia.

Escobar, Cristina. 2005. "La doble ciudadanía y la participación política: inmigrantes en la interacción de la política de Estados Unidos y Colombia”. Análisis Político 53: 3-21.

Estrada, Fernando. 2006. "Estado mínimo, agencias de protección y control territorial". Análisis Politico 56: 115-131.

García, Mauricio. 2005. "Repertorio de acciones colectivas en el movimiento por la paz en Colombia, 1978-2003”. Controversia 184: 149-173.

García, Mauricio. 2006a. "De Turbay a Uribe: sin política de paz pero con conflicto armado”. En Leal Buitrago, Fernando, En la encrucijada, Colombia en el siglo XXI. Bogotá: Norma, 479-512.

García, Mauricio. 2006b. Movimiento por la paz en Colombia, 1978-2003. Bogotá: CineP.

García-Peña, Daniel. 2005. "La relación del Estado colombiano con el fenómeno paramilitar: por el esclarecimiento histórico". Análisis Político 53: 58-76.

Gouëset, Vincent; Cuervo, Luis Mauricio; Lulle, Thierry; Coing, Henri. 2006. Hacer metrópoli. La región urbana de Bogotá de cara al siglo XXI. Bogotá: Universidad Externado de Colombia.

Gutiérrez Sanín, Francisco. 2006. "Estrenando sistema de partidos". Análisis Político 57: 106-125.

Gutiérrez Sanín, Francisco; Barón, Mauricio. 2006. "Estado, control territorial paramilitar y orden político en Colombia”. En Gutiérrez Sanín, Francisco; Sánchez, Gonzalo, Nuestra guerra sin nombre. Bogotá: Norma, 267-312.

Gutiérrez Sanín, Francisco; Sánchez, Gonzalo. 2006. Nuestra guerra sin nombre. Bogotá: Norma.

Guzmán Campos, Germán; Fals Borda, Orlando; Umaña luna, Eduardo. 1962. La violencia en Colombia. Bogotá: Tercer Mundo.

Hoskin, Gary; Masias, Rodolfo; Galvis, Marcela. 2005. "Modelos de decisión electoral y perfil de votante en Colombia: elecciones presidenciales 2002". Análisis Político 55: $60-74$. 
Hoyos, Diana. 2006. "Estabilidad del sistema de partidos en Colombia 1972-2000, una mirada local y regional". Análisis Político 55: 45-59.

Leal Butrago, Francisco. 2005. "La política de seguridad democrática: 2002-2005". Análisis Político 57: 3-30.

Leal Butrago, Francisco. 2006a. En la encrucijada, Colombia en el siglo XXI. Bogotá: Norma.

Leal Butrago, Francisco. 2006b. "Políticas de seguridad: de improvisación en improvisación”. En Leal Buitrago, Francisco (coordinador). 2006a, En la encrucijada, Colombia en el siglo XXI. Bogotá: Norma, 513-544.

Leal Buitrago, Francisco. 2006c. La inseguridad de la seguridad: Colombia 1958-2005. Bogotá: Planeta.

Leal Buttrago, Francisco; Rey, Germán. 2000. Discurso y razón. Una historia de las ciencias sociales en Colombia. Bogotá: Tercer Mundo.

Massal, Julie. 2005. "Nadando contra la corriente: mujeres y cuotas políticas en los países andinos". Análisis Político 55: 75-79.

Massal, Julie. 2006. "La reforma política en Ecuador, un callejón sin salida". Análisis Político 56: 132-150.

Medellín, Pedro. 2006. El Presidente sitiado: ingobernabilidad y erosión del poder presidencial en Colombia. Bogotá: Planeta.

MedinA, CARLOS. 2005. "La economía de guerra paramilitar: una aproximación a sus fuentes de financiamiento". Análisis político 53: 77-87.

Ortiz, Román. 2006. "La guerrilla mutante". En Leal Buitrago, Fernando. 2006a, En la encrucijada, Colombia en el siglo XXI. Bogotá: Norma, 323-356.

PARdo, Rodrigo. 2006. "Un país problema en un mundo intervencionista". En Leal Buitrago, Fernando. 2006a, En la encrucijada, Colombia en el siglo XXI. Bogotá: Norma, 545-570.

PéCAut, Daniel. 1988. Crónica de dos décadas de política colombiana. Bogotá: Siglo XXI.

PÉCAUT, DANIEL. 1998. "La contribución del IEPRI a los estudios sobre la violencia en Colombia”. Análisis politico 34: 71-89.

Pécaut, Daniel. 2006. Crónica de cuatro décadas de política colombiana. Bogotá: Norma.

Pizarro, Eduardo. 2006. "Las FARC-EP, ¿repliegue estratégico, debilitamiento o punto de inflexión?”. En Gutiérrez Sanín, Fernando; Sánchez, Gonzalo, Nuestra guerra sin nombre. Bogotá: Norma, 171-208.

RAMírez, SoCORRo. 2004. Intervención en conflictos interno. El Caso colombiano, 1994-2003. Bogotá: Universidad Nacional.

RAmírez, Socorro. 2006a. "Actores europeos ante el conflicto colombiano". En Gutiérrez Sanín, Fernando; Sánchez, Gonzalo, Nuestra guerra sin nombre. Bogotá: Norma, 71120.

RAmírez, Socorro. 2006b. "La ambigua regionalización del conflicto colombiano". En Gutiérrez Sanín, Fernando; Sánchez, Gonzalo, Nuestra guerra sin nombre. Bogotá: Norma, 121-169.

Ramírez, Socorro. 2006c. "El gobierno de Uribe y los países vecinos". Análisis político 57: 55-78.

Ramírez, Socorro; Cárdenas, José María. 2005. Venezuela y Colombia, debates de la historia y retos del presente. Bogotá: Universidad Nacional de Colombia, Caracas: Universidad Central de Venezuela.

Ramírez, Socorro; Cepik, Marcos. 2005. Agenda de seguridad andino-brasileña. Bogotá: FESCOL-IEPRI.

Rangel, Alfredo. 2005. El poder paramilitar. Bogotá: Planeta, Fundación Seguridad y democracia. 
Rojas, Diana. 2006a. "Estados Unidos y la guerra en Colombia". En Gutiérrez Sanín, Fernando; Sánchez, Gonzalo, Nuestra guerra sin nombre. Bogotá: Norma, 37-70.

Rojas, Diana. 2006b. "Balance de la política internacional del gobierno Uribe". Análisis político 57: 80-105.

Romero, MaUricio. 2006. "Paramilitares, narcotráfico y contrainsurgencia: una experiencia para no repetir”. En Gutiérrez Sanín, Fernando; Sánchez, Gonzalo, Nuestra guerra sin nombre. Bogotá: Norma, 357-386.

Schlenker, Juana; Iturralde, Manuel. 2006. "El uso del discurso de los derechos humanos por parte de los actores armados en Colombia: humanización del conflicto o estrategia de guerra". Análisis Político 56: 29-50.

Theidon, Kimberley; Betancourt, Paola Andrea. 2006. "Transiciones conflictivas, combatientes desmovilizados en Colombia”. Análisis Político 58: 92-111.

Thoumi, Francisco. 2005. "Ventajas competitivas ilegales, el desarrollo de la industria de drogas ilegales y el fracaso de las políticas contra las drogas en Afganistán y Colombia”. Análisis Político 54: 30-48.

Tickner, Arlene. 2006. "La securitización de la crisis colombiana: bases conceptuales y tendencias generales". Colombia Internacional 60: 90-112.

VARGAS, RicARDO. 2006. Narcotráfico, guerra y politica antidrogas: una perspectiva sobre las drogas en el conflicto armado colombiano. Bogotá: Novib, Transnational Institute.

Wills, María EMma. 2005a. “'Mujeres en armas: avance ciudadano o subyugación femenina?". Análisis Político 54: 63-80.

Wills, María Emma. 2005b. "Cincuenta años del sufragio femenino en Colombia, 1954: por la conquista del voto, 2004, por la ampliación de la ciudadanía de las mujeres". Análisis Político 53: 39-57.

Wills, María Emma; Gómez Correal, Diana. 2006. "Los movimientos sociales de mujeres (1970-2005); innovaciones, estancamientos y nuevas apuestas”. En Buitrago Real, Fernando, En la encrucijada, Colombia en el siglo XXI. Bogotá: Norma, 291-322. 